\title{
The Relationship Between Mean Platelet Volume and Fasting Plasma Glucose and HbA1c Levels in a Large Cohort of Unselected Health Check-Up Participants
}

\author{
Shinichiro Oshima ${ }^{a}$, Takakazu Higuchi ${ }^{b, c, e}$, Sadamu Okada ${ }^{b}$, Osamu Takahashi ${ }^{\mathrm{d}}$
}

\begin{abstract}
Background: Larger platelets are more active and mean platelet volume (MPV) is an indicator of platelet activation and an independent risk factor of cardiovascular diseases. While MPV is reported to be higher in diabetic patients, the relationship between MPV and glycemic parameters in general population remains inconclusive.
\end{abstract}

Methods: In this cross-sectional study, we studied relationship between MPV and fasting plasma glucose (FPG) and HbA1c levels in 38,204 unselected participants of general health check-up aged 20 years or older in the year of 2014 who were considered to be representative of the general population. Individuals with known diabetes, coronary artery disease and/or cerebrovascular disease who were on drug therapy and those with platelet counts below $100 \times 10^{9} / \mathrm{L}$ or above $400 \times 10^{9} / \mathrm{L}$ were excluded.

Results: The mean age of the individuals was $52.3 \pm 12.1$ years and $46.1 \%$ were male. There were positive associations between MPV and both FPG $(\mathrm{r}=0.066 ; \mathrm{P}<0.001)$ and HbA1c $(\mathrm{r}=0.025 ; \mathrm{P}<0.001)$ levels when all individuals were analyzed as a whole. While the association was only marginal in individuals with $\mathrm{HbAlc}$ levels below $6.5 \%(\mathrm{r}=0.009 ; \mathrm{P}=0.068)$, it was significant in those with $\mathrm{HbA} 1 \mathrm{c}$ $\geq 6.5 \%(\mathrm{r}=0.138 ; \mathrm{P}<0.001)$. When the individuals were categorized into four groups according to the $\mathrm{HbA} 1 \mathrm{c}$ values: $\mathrm{HbA} 1 \mathrm{c}<5.5 \%$, $5.5 \% \leq \mathrm{HbA} 1 \mathrm{c}<6.0 \%, 6.0 \% \leq \mathrm{HbA} 1 \mathrm{c}<6.5 \%$, and $\mathrm{HbA} 1 \mathrm{c} \geq 6.5 \%$, the mean MPV was virtually same among groups with HbAlc level $<$ $6.5 \%$ and that of the individuals with $\mathrm{HbA} 1 \mathrm{c} \geq 6.5$ was significantly higher than groups with lower HbA1c levels. Multivariate analyses adjusted with age and sex showed the same results.

Manuscript submitted January 15, 2018, accepted February 6, 2018

a Department of Internal Medicine, St. Luke's International Hospital, 9-1, Akashi-cho, Chuo-ku, Tokyo 104-8560, Japan

bDivision of Hematology, St. Luke's International Hospital, 9-1, Akashi-cho, Chuo-ku, Tokyo 104-8560, Japan

'Dokkyo Medical University Saitama Medical Center, 2-1-50, Minami-Koshigaya, Koshigaya 343-8555, Japan

${ }^{\mathrm{d}}$ Center for Clinical Epidemiology, St. Luke's International Hospital, 9-1, Akashi-cho, Chuo-ku, Tokyo 104-8560, Japan

${ }^{e}$ Corresponding Author: Takakazu Higuchi, Dokkyo Medical University Saitama Medical Center, 2-1-50, Minami-Koshigaya, Koshigaya 343-8555, Japan. Email: thiguchi@dokkyomed.ac.jp

doi: https://doi.org/10.14740/jocmr3361w
Conclusion: FPG and HbA1c appeared to be associated with MPV in unselected health check-up participants; however, the association between glycemic state and MPV was apparent only in individuals with impaired glycemic control and only marginal in those with normal glycemic control. The clinical significance of the associations warrants further study.

Keywords: Mean platelet volume; Diabetes; HbAlc; Fasting plasma glucose; Platelet activation; Thrombosis

\section{Introduction}

Cardiovascular disease is one of the major complications in patients with diabetes mellitus (DM). Diabetic patients are known to be in a prothrombotic state and at an increased risk of macrovascular complications such as coronary artery, cerebrovascular, and peripheral vascular diseases. Multiple mechanisms underlie the prothrombotic state and, among them, platelet activation is considered to play an important role in the pathogenesis of thrombotic complications in patients with DM $[1,2]$. A number of factors are reported to be associated with hyperactive platelets in patients with DM [3-6] and the size of platelets is considered to be involved in the activation, larger platelets being more active [6].

Mean platelet volume (MPV) is an indicator of platelet size which is determined automatically by automated blood cell counters and is an easy and inexpensive parameter of activation of platelets $[2,7,8]$. Platelet activation is well known to be very important among various pathophysiological mechanisms of atherosclerosis and there is an association between higher MPV value and coronary artery and cerebrovascular diseases and higher MPV is reported to be an independent risk factor of cardiovascular events [9-11].

It is well known that high blood glucose level is an independent risk factor of cardiovascular event. The size of platelets determined by MPV is reported to be larger in patients with DM [12-17] and may contribute to the prothrombotic state in patients with DM [1]. However, the relationship between MPV and glycemic parameters in non-diabetic individuals remains inconclusive and it is not yet known whether there is a true physiological association between platelet size and glycemic state in general population $[18,19]$. We conducted this cross- 


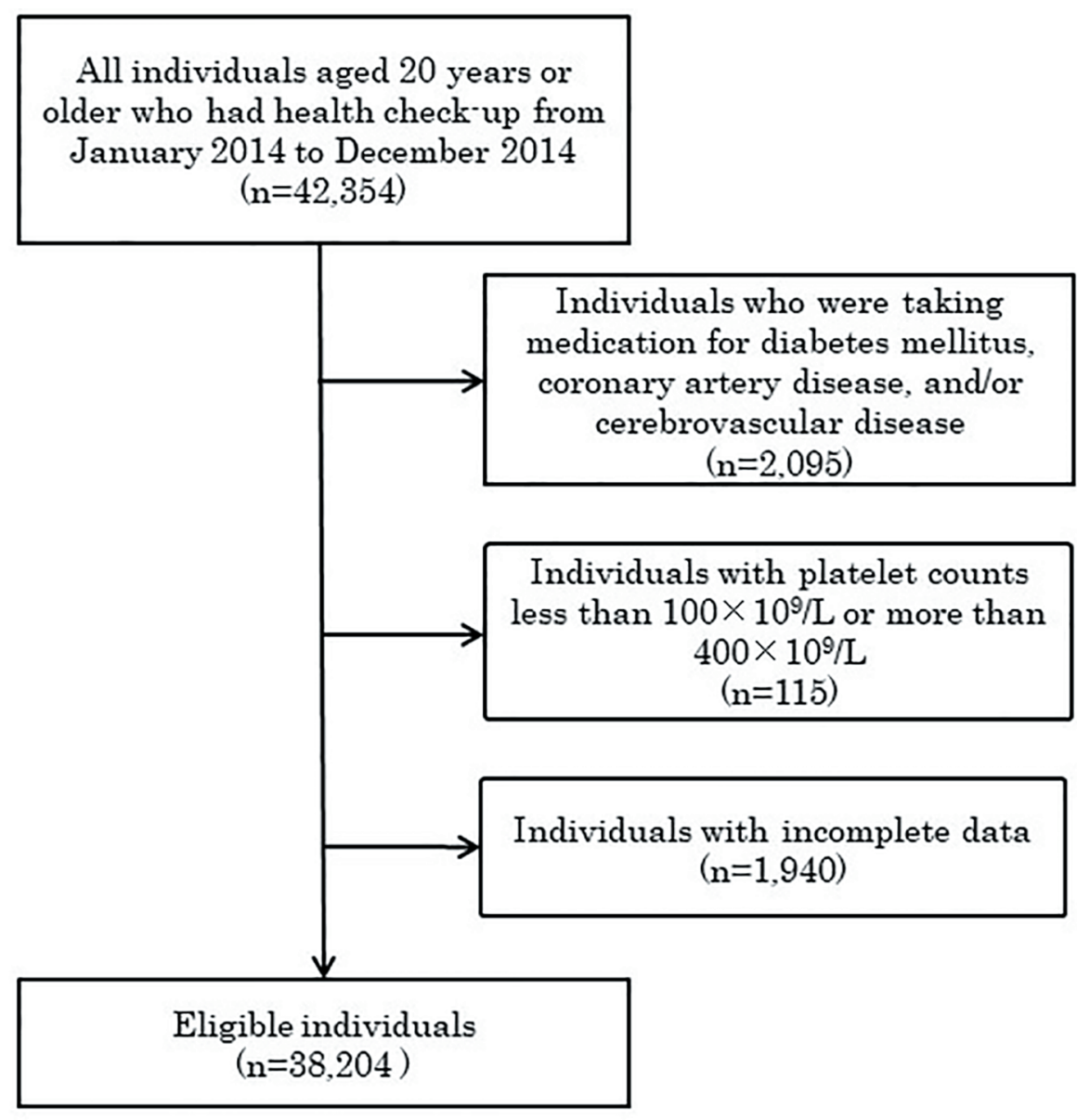

Figure 1. Study flow diagram.

sectional study of a large cohort of unselected participants of general health check-up to clarify the physiological association. If such association exists, it might be applied to detect the individual with higher risk of developing cardiovascular events by MPV and to allow early intervention and/or preventive measures.

\section{Methods}

This study is a retrospective observational study. All the individuals had approved the use of their data and The Ethics Committee of St. Luke's International Hospital approved the use of their clinical data.

\section{Study population and data collection}

We performed a retrospective search on the electronic database of all individuals aged 20 years or older who had health check-up at the Center for Preventive Medicine affiliated to St. Luke's International Hospital, Tokyo, Japan from January 2014 to December 2014. Exclusion criteria were patients who were taking medication including insulins for DM, coronary artery disease, and/or cerebrovascular disease including transient ischemic attack. In addition, subjects with platelet counts less than $100 \times 10^{9} / \mathrm{L}$ or more than $400 \times 10^{9} / \mathrm{L}$ were excluded to exclude subjects with possible hematological diseases which could affect MPV. For the individuals who had more than one health check-ups in 2014, only the results of the first checkup were studied. The medical information and laboratory data were collected by reviewing the electronic medical records. In all subjects, the blood samples were analyzed within $15 \mathrm{~min}$ after blood collection and complete blood cell count and MPV were analyzed with Sysmex XE-2100 automated cell counter (Sysmex Corp., Japan).

\section{Statistical analysis}

Statistical analyses were performed using the IBM SPSS ver. 22 (Tokyo, Japan). The comparisons among groups were performed using ANOVA for continuous variables with Bonferroni correction when appropriate. Pearson's correlation coefficients were calculated to evaluate the relationship between MPV and fasting plasma glucose (FPG) and HbAlc. As this study was not intended to compare MPV values according to their glucose tolerance status, subjects were stratified based on 
Table 1. Characteristic of the Entire Subjects $(n=38,204)$

\begin{tabular}{ll}
\hline Characteristics & \\
\hline Age, mean (SD), years & $52.3(12.1)$ \\
Male, n (\%) & $17,622(46.1)$ \\
FPG, mean (SD), mg/dL & $98.9(11.3)$ \\
HbAlc, mean (SD), \% & $5.5(0.4)$ \\
MPV, mean (SD), fL & $9.97(0.71)$ \\
\hline
\end{tabular}

FPG: fasting plasma glucose; MPV: mean platelet volume.

the $\mathrm{HbA} 1 \mathrm{c}$ into the following four groups: group 1 (HbA1c $<5.5 \%)$, group $2(5.5 \% \leq \mathrm{HbA} 1 \mathrm{c}<6.0 \%)$, group $3(6.0 \% \leq$ $\mathrm{HbA} 1 \mathrm{c}<6.5 \%)$, and group 4 (HbA1c $\geq 6.5 \%)$, and analyzed with MPV as the outcome using multiple linear regression analysis and adjusted with age and sex. All analyses were two tailed and $\mathrm{P}$ values below 0.05 were considered to be statistically significant.

\section{Results}

Forty-two thousand three hundred fifty-four subjects had general health check-up from January 2014 to December 2014 (Fig. 1). Out of them, 2,095 individuals who were taking medication for DM, coronary artery disease, and/or cerebrovascular disease were excluded. One hundred and fifteen and 1,940 individuals were also excluded due to the platelet count less than $100 \times 10^{9} / \mathrm{L}$ or more than $400 \times 10^{9} / \mathrm{L}$ and incomplete data, respectively. As a result, 38,204 subjects were enrolled in this cross-sectional study.
The characteristics of the 38,204 subjects included in the analyses are presented in Table 1. The mean age was 52.3 (SD: 12.1) years and $17,622(46.1 \%)$ were male. The mean FPG level was $98.9 \pm 11.3 \mathrm{mg} / \mathrm{dL}$ and the mean HbA1c level was $5.5 \pm 0.4 \%$ and, thus, it was obvious that the study population included a considerable number of individuals with overt DM or impaired glucose tolerance who had been treated with nondrug therapy or who had not been under medical attention before (Figs. 2 and 3). The mean MPV of the entire subjects was $9.97 \pm 0.71 \mathrm{fL}$.

We first studied the correlations between indicators of glycemic status and MPV in the whole subjects. Positive correlations were found between MPV and both FPG ( $\mathrm{r}=0.066$, $\mathrm{P}<0.001)$ (Fig. 2) and HbA1c $(\mathrm{r}=0.025, \mathrm{P}<0.001)$ (Fig. 3) levels when all subjects were analyzed as a whole, indicating that there are linear correlations between both FPG and HbA1c levels and MPV in unselected individuals.

We next stratified the subjects into four groups according to the HbAlc value and compared the mean MPV of the groups (Table 2). A total of 15,974 subjects were stratified in group 1 (HbA1c $<5.5 \%), 18,044$ in group $2(5.5 \% \leq \mathrm{HbA} 1 \mathrm{c}$ $<6.0 \%), 3,529$ in group $3(6.0 \% \leq \mathrm{HbA} 1 \mathrm{c}<6.5 \%)$, and 657 in group $4(\mathrm{HbA} 1 \mathrm{c} \geq 6.5 \%)$. The mean age of the groups with higher HbA1c level was significantly older and the groups with higher HbA1c level included significantly more male than the lower HbA1c groups ( $\mathrm{P}<0.001$ for both characteristics). The mean MPV value in group 2 was $0.004 \mathrm{fL}$ larger than that in group 1; however, statistically they were virtually the same (P = 1.0) (Fig. 4). Thereafter, the mean MPV value appeared to increase in the groups with higher HbA1c value; however, the increase was not significant among individuals in non-diabetic range, the increases in the mean MPV between group 3 and

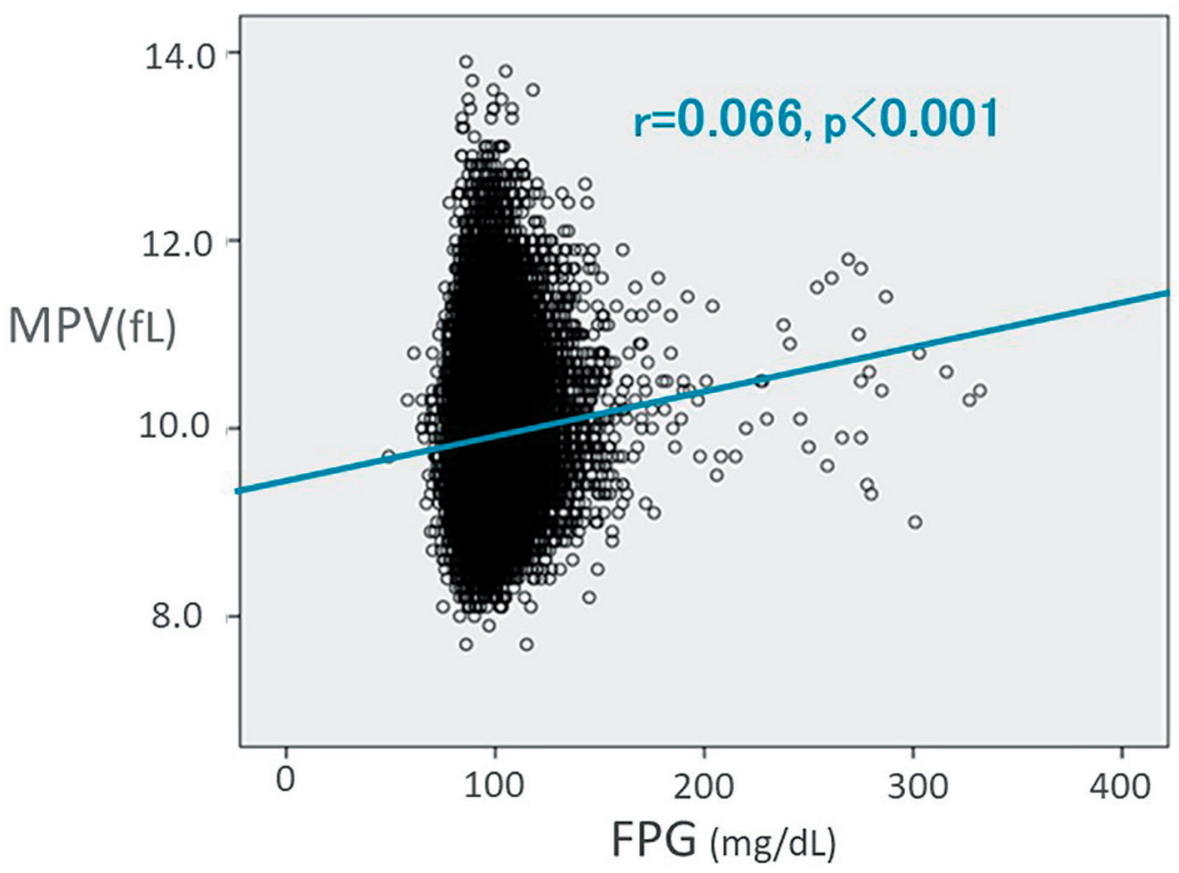

Figure 2. Pearson's correlation showing a linear relation between mean platelet volume (MPV) and fasting plasma glucose (FPG). 


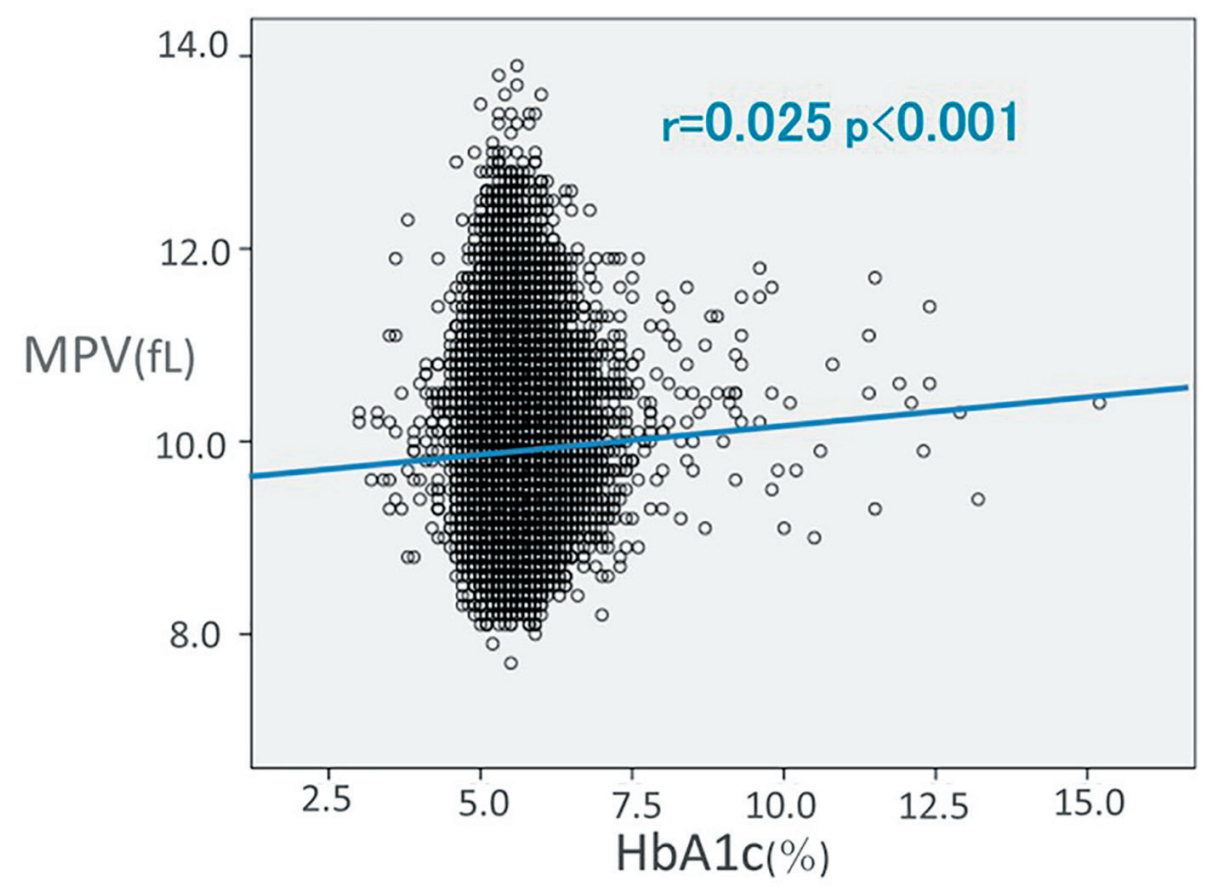

Figure 3. Pearson's correlation showing a linear relation between mean platelet volume (MPV) and HbA1c.

group 1 being $0.025 \mathrm{fL}(\mathrm{P}=0.313)$. The mean MPV of group 4 was $0.125 \mathrm{fL}$ larger than group $1(\mathrm{P}<0.001)$, showing a significant increase in MPV value in the group with $\mathrm{HbA} 1 \mathrm{c}$ level in diabetic range compared to the group with the lowest $\mathrm{HbA} 1 \mathrm{c}$ level. When the mean MPV values of each two groups were analyzed with Bonferroni correction, the difference was significant only between group 4 and either of groups 1, 2, and 3 , with $\mathrm{P}$ values being $<0.001,<0.001$, and 0.006 for each comparison.

Based on these findings, we analyzed the correlation between $\mathrm{HbAlc}$ and MPV levels in individuals with HbAlc levels below $6.5 \%$ and found that the correlation was only marginal in non-diabetic individuals $(\mathrm{r}=0.009 ; \mathrm{P}=0.068)$. On the other hand, the correlation was significant in individuals with $\mathrm{HbAlc} \geq 6.5 \%(\mathrm{r}=0.138 ; \mathrm{P}<0.001)$. These findings suggested that, while MPV appeared to be positively associated with glycemic state, the association was not apparent in nondiabetic individuals and was evident only in individuals with impaired glycemic control.

\section{Discussion}

Macrovascular complications are major causes of death in patients with DM and of the utmost importance in the management of DM. While multiple factors such as endothelial dysfunction and activation of coagulation system contribute to the prothrombotic state in diabetic patients [1], activated platelets play an essential role in the development of macrovascular complications [3-6]. Platelets are activated in patients with $\mathrm{DM}$ by various mechanisms such as increased platelet surface expression of glycoproteins $\mathrm{Ib}$ and IIb-IIIa, increased production of reactive oxygen species and thromboxane A2, altered calcium homeostasis, increase in tyrosine phosphorylation, and impaired generation of nitric oxide [3-6]. In addition, although some reports argue against the notion [20, 21], most re-

Table 2. Characteristics and Laboratory Data of the Subjects Categorized According to HbA1c Value

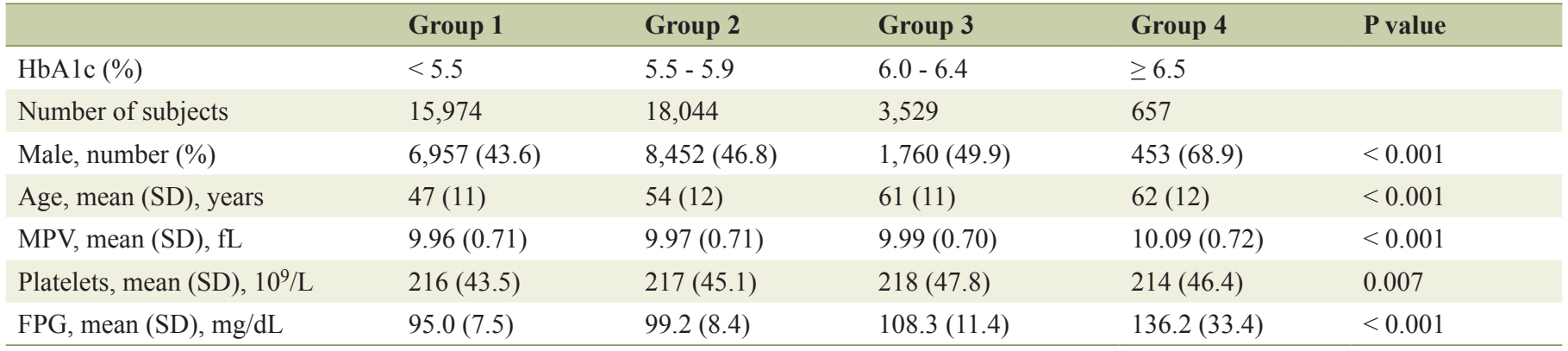

MPV: mean platelet volume; FPG: fasting plasma glucose. 


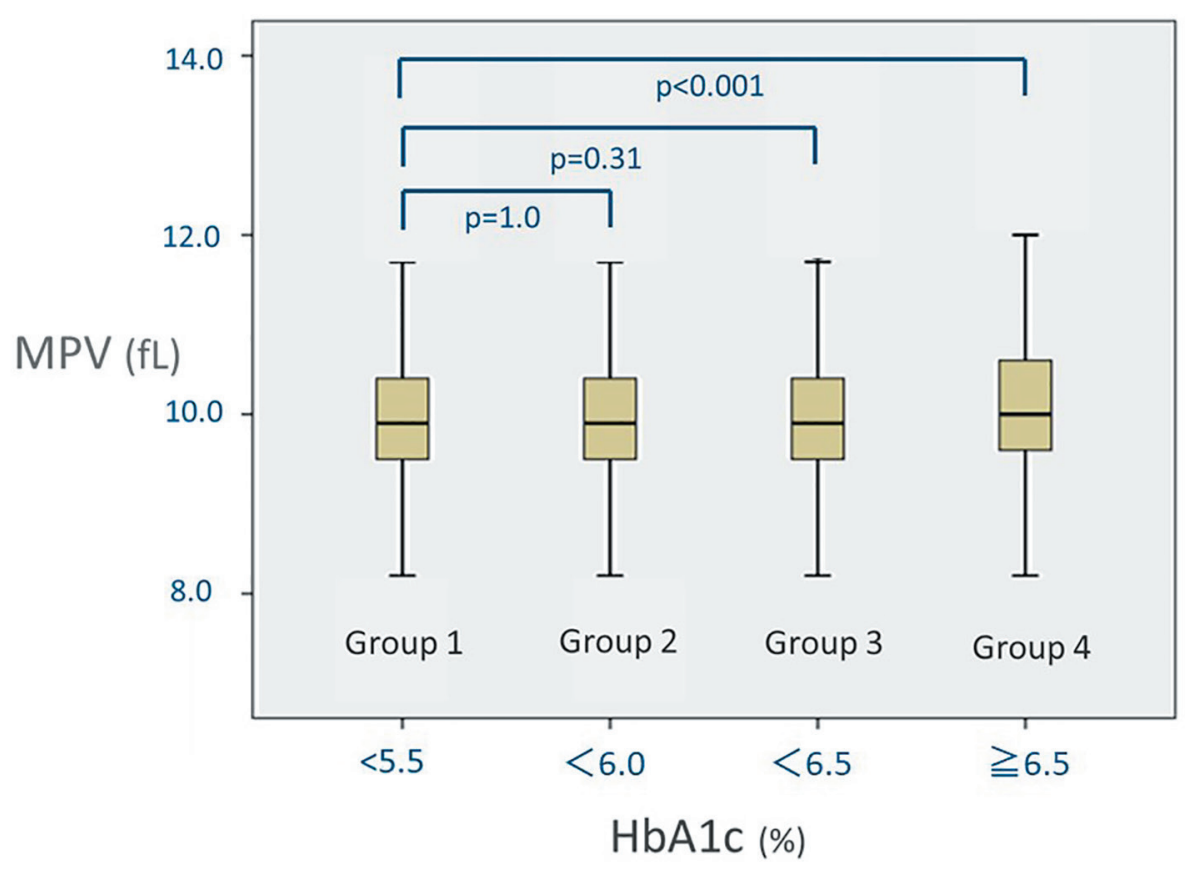

Figure 4. Distribution of mean platelet volume (MPV) in groups stratified according to the HbA1c values. Box-whisker plots of MPV in each group categorized by HbA1c value: group 1 (HbA1c $<5.5 \%)$, group $2(5.5 \% \leq \mathrm{HbA} 1 \mathrm{c}<5.9 \%)$, group $3(6.0 \% \leq$ $\mathrm{HbA} 1 \mathrm{c}<6.5 \%)$, and group $4(\mathrm{HbA} 1 \mathrm{c} \geq 6.5 \%)$, are presented. Lines indicate interquartile range.

ports have noted that the size of the platelets as determined by MPV is higher in patients with DM [12-17] and also in patients with impaired glucose tolerance $[15,22]$ than normal subjects and associated with platelet activation. MPV is an indicator of platelet size and large platelets are more active physiologically and enzymatically [2]. Larger platelets contain more granules and produce greater amounts of vasoactive and thrombotic factors, possess greater aggregability in response to stimulus, and express a greater number of adhesion molecules [8]. These findings indicate the contribution of larger activated platelets in the development of thrombotic complications in diabetic patients. Also, clinical relevance of MPV is demonstrated by the observations that MPV is higher in patients with coronary artery disease and associated with prognosis $[9,10,16]$. As the size of platelets is considered to remain fairly constant during their life-span $[2,23]$, the mechanism of the increased platelet volume in diabetic patients is not fully clarified, although osmotic swelling of platelet induced by hyperglycemia [24] and accelerated platelet turnover [1] are considered to be attributed.

If there is a true physiological association between indicators of glycemic control and MPV, the association should be observed in a general population as well and, if there is an association, it would be possible to detect individuals in both diabetic and non-diabetic populations with activated platelets easily and without additional costs and to intervene to prevent future thrombotic events. Thus, we studied a large cohort of individuals who were considered to be representative of general population using HbA1c and FPG as indicators of the glycemic state.

Studies on the association between glycemic status and
MPV in general population are limited and included smaller number of participants of health check-ups with inconsistent results $[18,19,25]$. One report studied 1,876 subjects and found that MPV was positively associated with FPG level [25]. Another studied 4,072 subjects and found a positive association between MPV and HbA1c level [19]. However, the third report by Kim et al which studied 3,098 individuals was against such associations [18]. They found a positive correlation between MPV and FPG level only in diabetic women but not in individuals with normal glucose tolerance and intermediate hyperglycemia. We did not subdivide individuals into groups according to their glucose tolerance status but studied the association simply based on the laboratory values as continuous variables because our aim was to study the physiological association between glycemic status and MPV in general population. Thus, we could not determine the difference according to their diagnosis, such as impaired glucose tolerance and diabetes. Nonetheless, our study involved far larger number of individuals from general population and found that, while there appeared to be some physiological correlation between glycemic control and MPV in individuals as a whole, the correlation was very weak in non-diabetic range and was significant only in individuals with impaired glycemic control.

If there is a physiological association between the indicators of glycemic state and MPV, the clinical relevance of MPV in general population does not appear very high at present because of the wide variation of MPV value at given levels of FPG and HbAlc compared to the normal reference range of MPV: 7.7 - 13.9 fL, and also of the modest difference of 0.125 $\mathrm{fL}$ in the mean MPV values between the highest and lowest $\mathrm{HbA} 1 \mathrm{c}$ groups. 
Other limitations of this study are that this is a cross-sectional study and the outcome is not the incidence of cardiovascular event. Therefore, prospective studies to evaluate whether the hyperglycemic status increases MPV continuously and the decrease in MPV value is associated with the reduction in the risk of developing cardiovascular events are necessary to determine the clinical significance of MPV.

Nonetheless, this study demonstrated that parameters of glycemic control is associated with MPV at least in unselected general population with unrecognized or untreated impaired glycemic state and the clinical significance of the association warrants further study.

\section{References}

1. Vazzana N, Ranalli P, Cuccurullo C, Davi G. Diabetes mellitus and thrombosis. Thromb Res. 2012;129(3):371377.

2. Gasparyan AY, Ayvazyan L, Mikhailidis DP, Kitas GD. Mean platelet volume: a link between thrombosis and inflammation? Curr Pharmaceut Design. 2011;17(1):47-58.

3. Sobol AB, Watala C. The role of platelets in diabetesrelated vascular complications. Diabet Res Clin Pract. 2000;50(1):1-16.

4. Vinik AI, Erbas T, Park TS, Nolan R, Pittenger GL. Platelet dysfunction in type 2 diabetes. Diabetes Care. 2001;24(8):1476-1485.

5. El Haouari M, Rosado JA. Platelet signalling abnormalities in patients with type 2 diabetes mellitus: a review. Blood Cells Mol Dis. 2008;41(1):119-123.

6. Morel O, Kessler L, Ohlmann P, Bareiss P. Diabetes and the platelet: toward new therapeutic paradigms for diabetic atherothrombosis. Atherosclerosis. 2010;212(2):367376.

7. Tsiara S, Elisaf M, Jagroop IA, Mikhailidis DP. Platelets as predictors of vascular risk: Is there a practical index of platelet activity? Clin Appl Thrombosis/Hemostasis. 2003;9(3):177-190.

8. Vizioli L, Muscari S, Muscari A. The relationship of mean platelet volume with the risk and prognosis of cardiovascular diseases. Int J Clin Pract. 2009;63(10):1509-1515.

9. Chu SG, Becker RC, Berger PB, Bhatt DL, Eikelboom JW, Konkle B, Mohler ER, et al. Mean platelet volume as a predictor of cardiovascular risk: a systematic review and meta-analysis. J Thromb Haemost. 2010;8(1):148156.

10. Sansanayudh N, Anothaisintawee T, Muntham D, McEvoy M, Attia J, Thakkinstian A. Mean platelet volume and coronary artery disease: a systematic review and meta-analysis. Int J Cardiol. 2014;175(3):433-440.

11. Han JY, Choi DH, Choi SW, Kim BB, Ki YJ, Chung JW, Koh YY, et al. Stroke or coronary artery disease prediction from mean platelet volume in patients with type 2 diabetes mellitus. Platelets. 2013;24(5):401-406.

12. Lee EY, Kim SJ, Song YJ, Choi SJ, Song J. Immature platelet fraction in diabetes mellitus and metabolic syndrome. Thromb Res. 2013;132(6):692-695.
13. Kodiatte TA, Manikyam UK, Rao SB, Jagadish TM, Reddy M, Lingaiah HKM, Lakshmaiah V. Mean platelet volume in Type 2 diabetes mellitus. J Lab Physicians. 2012;4(1):5-9.

14. Papanas N, Symeonidis G, Maltezos E, Mavridis G, Karavageli E, Vosnakidis T, Lakasas G. Mean platelet volume in patients with type 2 diabetes mellitus. Platelets. 2004;15(8):475-478.

15. Ozder A, Eker HH. Investigation of mean platelet volume in patients with type 2 diabetes mellitus and in subjects with impaired fasting glucose: a cost-effective tool in primary health care? Int J Clin Exp Med. 2014;7(8):22922297.

16. Lekston A, Hudzik B, Hawranek M, Szkodzinski J, Gorol J, Wilczek K, Gasior M, et al. Prognostic significance of mean platelet volume in diabetic patients with ST-elevation myocardial infarction. J Diabetes Complications. 2014;28(5):652-657.

17. Kim ES, Mo EY, Moon SD, Han JH. Mean platelet volume is closely associated with serum glucose level but not with arterial stiffness and carotid atherosclerosis in patients with type 2 diabetes. J Clin Endocrinol Metab. 2015;100(9):3502-3508.

18. Kim JH, Kang SB, Kang JI, Kim JW, Kim SY, Bae HY. The relationship between mean platelet volume and fasting plasma glucose differs with glucose tolerance status in a Korean general population: gender differences. Platelets. 2013;24(6):469-473.

19. Lippi G, Salvagno GL, Nouvenne A, Meschi T, Borghi $\mathrm{L}$, Targher $\mathrm{G}$. The mean platelet volume is significantly associated with higher glycated hemoglobin in a large population of unselected outpatients. Prim Care Diabetes. 2015;9(3):226-230.

20. Unubol M, Ayhan M, Guney E. The relationship between mean platelet volume with microalbuminuria and glycemic control in patients with type II diabetes mellitus. Platelets. 2012;23(6):475-480.

21. Verdoia M, Schaffer A, Barbieri L, Cassetti E, Nardin M, Bellomo G, Marino P, et al. Diabetes, glucose control and mean platelet volume: a single-centre cohort study. Diabetes Res Clin Pract. 2014;104(2):288-294.

22. Coban E, Kucuktag S, Basyigit S. Platelet activation in subjects with impaired glucose tolerance. Platelets. 2007;18(8):591-594.

23. Bavbek N, Kargili A, Kaftan O, Karakurt F, Kosar A, Akcay A. Elevated concentrations of soluble adhesion molecules and large platelets in diabetic patients: are they markers of vascular disease and diabetic nephropathy? Clin Appl Thromb Hemost. 2007;13(4):391-397.

24. Martyn CN, Matthews DM, Popp-Snijders C, Tucker J, Ewing DJ, Clarke BF. Effects of sorbinil treatment on erythrocytes and platelets of persons with diabetes. Diabetes Care. 1986;9(1):36-39.

25. Shimodaira M, Niwa T, Nakajima K, Kobayashi M, Hanyu N, Nakayama T. Correlation between mean platelet volume and fasting plasma glucose levels in prediabetic and normoglycemic individuals. Cardiovasc Diabetol. 2013;12:14. 